\title{
A norm compatible system of Galois cohomology classes for $\operatorname{GSp}(4)$
}

Francesco Lemma

\begin{abstract}
For a given de Rham $p$-adic Galois representation $M$, a conjecture of Perrin-Riou associates a $p$-adic $L$-function for $M$ to a norm compatible system of Galois cohomology classes in the projective limit $\lim _{n} \mathrm{H}^{1}\left(\mathbb{Q}\left(\zeta_{p^{n}}\right), M\right)$. We construct such a norm compatible system for the symplectic group $\overleftarrow{\mathrm{SSp}}_{4}$. Our classes are cup-products of torsion sections of the large elliptic polylogarithm pro-sheaf; we rely on its norm compatibility and on some computations of weights in the cohomology of Siegel threefolds of our previous work.
\end{abstract}

Mathematics Subject Classification (2010). 11M50, 11R23, 11F67.

Keywords. Elliptic polylogarithm pro-sheaf, $p$-adic $L$-function, Siegel modular variety.

\section{Introduction}

$L$-functions are defined as convergent infinite series of complex numbers and their values at integers have an algebraic meaning, like in the analytic class number formula of Dedekind and Dirichlet. In particular, it should be possible to find a $p$-adic analytic $L$-function taking the same values as the archimedean one at some integers. For example, consider two positive integers $a$ and $b$ prime to an odd prime number $p$, and fix a system of primitive $p^{n}$-th roots of unity $\zeta_{n}$, such that $\zeta_{n}^{p}=\zeta_{n-1}$. The numbers

$$
\frac{\zeta_{n}^{-a / 2}-\zeta_{n}^{a / 2}}{\zeta_{n}^{-b / 2}-\zeta_{n}^{b / 2}}
$$

are units of the rings of integers $\mathbb{Z}\left[\zeta_{n}\right]$ mapped to each other under the norms $\mathbb{Z}\left[\zeta_{m}\right]^{\times} \rightarrow \mathbb{Z}\left[\zeta_{n}\right]^{\times}$for $n \mid m$. We owe to Kubota-Leopold and Iwasawa that to this compatible system of units is associated a (pseudo-)measure $d \zeta_{p}$ on $\mathbb{Z}_{p}^{\times}$such that

$$
\int_{\mathbb{Z}_{p}^{\times}} x^{k} d \zeta_{p}=\left(b^{k}-a^{k}\right)\left(1-p^{k-1}\right) \zeta(1-k),
$$

for any even positive integer $k$, where $\zeta$ denotes the Riemann zeta function (see [5], 4.2 for details). Via the boundary map coming from the Kummer exact sequence, these 
units should be seen as a norm compatible system of Galois cohomology classes in the projective limit $\lim _{n} \mathrm{H}^{1}\left(\mathbb{Q}\left(\zeta_{n}\right), \mathbb{Z}_{p}(1)\right)$ associated to the Tate motive. Now given any de Rham $p$-adic Galois representation $M$, a conjecture of Perrin-Riou associates a $p$-adic $L$-function for $M$ to a compatible system of classes belonging to the inverse limit $\lim _{n} \mathrm{H}^{1}\left(\mathbb{Q}\left(\zeta_{p^{n}}\right), M\right)$, via $p$-adic Hodge theory and a $p$-adic interpolation of Bloch-Kato exponential maps ([17], Chapitre 4, Conjecture CP(M)).

Only few examples of such systems of cohomology classes are known: for example, there is the one described above, and the system of Beilinson-Kato's elements defined as $K$-theoretical cup-products of modular units, and giving rise to the $p$-adic $L$-function of elliptic modular forms ([10], Theorem 16.6(2)).

This note provides another example of such a norm compatible system for the symplectic group in four variables $\mathrm{GSp}_{4}$. The main ingredient is the norm compatibility of the large elliptic polylogarithm pro-sheaf, due to Wildeshaus, and the computations of weights in the cohomology of Siegel threefolds of our previous work [15], Section 2.2. Indeed, the cohomology classes considered here are the $p$-adic realization of cup-products of Beilinson's Eisenstein symbols (see [11], Theorem 3.2.1), which are torsion sections of the elliptic polylogarithm pro-sheaf. In fact, both the system of cyclotomic units described above and the one of modular units defined in [10], Section 2.2, can be seen as the $p$-adic realization of the torsion sections of the classical and elliptic polylogarithm respectively ([21], IV, Chapter 4, Theorem 4.5, and [12], Theorem 4.2.9).

In a forthcoming paper we expect to relate our system to the critical values of the degree four $L$-function of cuspidal automorphic representations of $\mathrm{GSp}_{4}$ as predicted by the conjecture of Perrin-Riou.

\section{Conventions and notations}

In this section we fix conventions and notations for the rest of the paper. We advise the reader to consult this section only according to his needs.

1.1. In this note, we consider a fixed prime number $p \geq 3$. Given a $\mathbb{Q}$-scheme of finite type $X$, we work in the setting of bounded derived categories $\mathrm{D}_{c}^{b}\left(X, \mathbb{Q}_{p}\right)$ of [7], where the coefficient ring is the field $\mathbb{Q}_{p}$. The category of smooth étale $\mathbb{Q}_{p}$-sheaves naturally embeds in the heart of $\mathrm{D}_{c}^{b}\left(X, \mathbb{Q}_{p}\right)$ and on the derived categories $\mathrm{D}_{c}^{b}\left(X, \mathbb{Q}_{p}\right)$ we have the formalism of Grothendieck's six functors $\left(f^{*}, f_{*}, f_{!}, f^{!}, \underline{\text { Hom }}, \hat{\otimes}\right)$.

1.2. The large elliptic polylogarithm is an extension of pro-sheaves. For a given abelian category $\mathcal{A}$, the category pro- $\mathcal{A}$ of pro-objects of $\mathcal{A}$ is the category whose objects are projective systems

$$
A=\left(A_{i}\right)_{i \in I}: I^{\mathrm{op}} \rightarrow \mathcal{A}
$$


where $I$ is some small filtered index category. The morphisms are

$$
\operatorname{Hom}_{\text {pro-AA }}\left(\left(A_{i}\right)_{i},\left(B_{j}\right)_{j}\right)=\lim _{j} \lim _{i} \operatorname{Hom}_{\mathcal{A}}\left(A_{i}, B_{j}\right)
$$

The category pro- $\mathcal{A}$ is again abelian ([1], A 4.5) and a functor $F: \mathcal{A} \rightarrow \mathcal{B}$ is extended to the pro-categories in the obvious way $F\left(\left(A_{i}\right)_{i}\right)=\left(F\left(A_{i}\right)\right)_{i}$. We are interested in the abelian category $\operatorname{Sh}_{\Lambda}(X)$ of étale sheaves of $\Lambda$-modules over a scheme $X$. Given two pro-sheaves $\left(A_{i}\right)$ and $\left(B_{i}\right)$, we denote by $\operatorname{Ext}_{X}^{j}\left(\left(A_{i}\right),\left(B_{i}\right)\right)$ the group of $j$-th Yoneda extensions of $\left(A_{i}\right)$ by $\left(B_{i}\right)$ in the category pro- $\mathcal{A}$.

1.3. Siegel threefolds. We fix a four-dimensional symplectic space $\left(V_{4}, \psi\right)$ over $\mathbb{Z}$ and denote by

$$
\begin{aligned}
\mathrm{G}=\mathrm{GSp}_{4}=\left\{g \in \mathrm{GL}\left(V_{4}\right) \mid \text { there exists } v(g) \in \mathbb{G}_{m}\right. \text { such that } \\
\qquad \begin{array}{l}
\left.\psi(g v, g w)=v(g) \psi(v, w) \text { for all } v, w \in V_{4}\right\}
\end{array}
\end{aligned}
$$

the associated symplectic group, with center $Z$ and derived group $\mathrm{Sp}_{4}=\operatorname{ker} v$. Denote by $K_{\infty}$ a maximal compact subgroup modulo the center $Z(\mathbb{R}) K_{\infty}^{\prime} \subset \mathrm{G}(\mathbb{R})$ where $K_{\infty}^{\prime}$ is a maximal compact subgroup of $\mathrm{Sp}_{4}(\mathbb{R})$. The locally compact topological ring of adeles of $\mathbb{Q}$ is $\mathbb{A}=\mathbb{R} \times \mathbb{A}_{f}$ where $\mathbb{A}_{f}=\mathbb{Q} \otimes \widehat{\mathbb{Z}}$ and $\widehat{\mathbb{Z}}=\lim _{N} \mathbb{Z} / N \mathbb{Z}$. For every non zero integer $N$ we consider the compact open subgroup $K(N) \subset \mathrm{G}\left(\mathbb{A}_{f}\right)$ kernel of the reduction $\mathrm{G}(\widehat{\mathbb{Z}}) \rightarrow \mathrm{G}(\mathbb{Z} / N \mathbb{Z})$ modulo $N$. Given a $\mathbb{Z}\left[\frac{1}{N}\right]$-scheme $S$ we consider the set of uples $\{A, \lambda, \zeta, \eta\}$ made of an abelian scheme $A \rightarrow S$ of relative dimension 2 , a principal polarisation $\lambda$, i.e., an isomorphism $\lambda: A \rightarrow \hat{A}$ with the dual abelian scheme and whose dual is $\lambda$ itself, a primitive $N$-th root of unity over $S$ and a principal level $N$ structure, i.e., an $S$-group schemes isomorphism $V_{4} / N V_{4} \otimes S \simeq A[N]$ with the $N$-torsion of $A$, compatible with $\psi$ and $\lambda$ in an obvious sense. For $N \geq 3$, by a theorem of Mumford (see [16], Chapter 7, Theorem 7.9), the functor $S \mapsto\{A, \lambda, \zeta, \eta\}$ is representable by a smooth and quasi-projective $\mathbb{Z}\left[\frac{1}{N}, \zeta_{N}\right]$-scheme $\mathrm{S}(N)$ of dimension 3 . Fixing a complex embedding of the abelian extension $\mathbb{Q}\left(\zeta_{N}\right)$ generated by $N$-th roots of unity, we have $\mathrm{S}(N)(\mathbb{C})=\mathrm{G}(\mathbb{Q}) \backslash\left(\mathrm{G}(\mathbb{A}) / K(N) K_{\infty}\right)([14]$, Corollary 3.3). In this note we work with a fixed complex embedding of the field $\mathbb{Q}\left(\zeta_{N}\right)$.

\section{The large elliptic polylogarithm: definition and basic properties}

Let us present the definition and the basic properties of the large elliptic polylogarithm pro-sheaf. The following introduction follows closely Section 3 in Kings [12].

In this section $S$ will denote a connected scheme of characteristic zero. By an elliptic curve over $S$, we mean a proper and smooth $S$-group scheme of relative dimension one. Let $\pi: E \rightarrow S$ be such a morphism, with unit section $e: S \rightarrow E$. 
Definition 2.1. A lisse $\mathbb{Q}_{p}$-sheaf $\mathscr{F}$ over $E$ is said to be $n$-unipotent of length $n$ if it admits a filtration $\mathscr{F}^{\prime}=\mathscr{F}^{0} \supset \mathscr{F}^{1} \supset \cdots \supset \mathscr{F}^{n} \supset \mathscr{F}^{n+1}=0$ such that $\operatorname{Gr}^{i} \mathscr{F}=\pi^{*} \mathscr{G}^{i}$ for some lisse $\mathbb{Q}_{p}$-sheaf $\mathscr{G}^{i}$ over $S$.

Theorem 2.2 ([3], Proposition 1.2.6). Up to unique isomorphism, there is a unique $n$-unipotent sheaf $\mathscr{L}_{E}^{(n)}$ of length $n$, together with a section of the fibre at the unit section $1^{(n)}: \mathbb{Q}_{p} \rightarrow e^{*} \mathscr{L}_{\mathrm{L}} g_{E}^{(n)}$, such that for every $n$-unipotent sheaf $\mathcal{F}$ the map $\pi_{*} \underline{\operatorname{Hom}}\left(\mathscr{L}^{\circ} g_{E}^{(n)}, \mathcal{F}\right) \rightarrow e^{*} \mathcal{F}$ mapping $f$ to $f \circ 1^{(n)}$ is an isomorphism.

Definition 2.3. The canonical maps $\log _{E}^{(n+1)} \rightarrow \mathscr{L}^{\circ} g_{E}^{(n)}$ that map $1^{(n+1)}$ to $1^{(n)}$ define the logarithm pro-sheaf

$$
\mathscr{L}_{0}=\left(\mathscr{L}_{\circ}(n)\right)_{E} .
$$

By the universal property of $\mathscr{L}^{\circ} g_{E}$, the pull-back $\mathcal{R}=e^{*} \mathscr{L}^{\circ} g_{E}$ is a ring with unit $\left(1^{(n)}\right)_{n}$ and the ring $\pi^{*} \mathcal{R}$ acts on $\mathscr{L}^{\circ} g_{E}$.

In order to give another very simple description of the logarithm pro-sheaf, consider the multiplication by $p^{j}$ which is an étale cover $\left[p^{j}\right]: E \rightarrow E$ over $S$ (recall that we assumed that $S$ is of characteristic zero). As $\left[p^{j}\right]$ is smooth, of relative dimension zero, we have $\left[p^{j}\right]^{!} \mathbb{Q}_{p}=\left[p^{j}\right]^{*} \mathbb{Q}_{p}=\mathbb{Q}_{p}$ and because $\left[p^{j}\right]$ is finite, hence proper, we have $\left[p^{j}\right]_{!}=\left[p^{j}\right]_{*}$ for every integer $j$. For $j^{\prime} \geq j$, we have $\left[p^{j^{\prime}}\right]=\left[p^{j}\right] \circ\left[p^{j^{\prime}-j}\right]$, hence the image of the counit $\left[p^{j^{\prime}-j}\right]_{!}\left[p^{j^{\prime}-j}\right]^{!} \mathbb{Q}_{p}=\left[p^{j^{\prime}-j}\right]_{*} \mathbb{Q}_{p} \rightarrow \mathbb{Q}_{p}$ under $\left[p^{j}\right]_{*}$ is a morphism $\operatorname{tr}_{j^{\prime} j}:\left[p^{j^{\prime}}\right]_{*} \mathbb{Q}_{p} \rightarrow\left[p^{j}\right]_{*} \mathbb{Q}_{p}$. By [12], Proposition 3.4.2 and Lemma 3.4.3, we have a canonical isomorphism of pro-sheaves

$$
\mathscr{L}_{E} \simeq\left(\left[p^{j}\right]_{*} \mathbb{Q}_{p}\right)_{j},
$$

where the transition maps on the right are given by the $\operatorname{tr}_{j^{\prime} j}$.

Now let $\mathcal{I}$ be the kernel of the augmentation map $\mathcal{R} \rightarrow \mathbb{Q}_{p}$. Denote by $j$ the open embedding complementary to the unit section

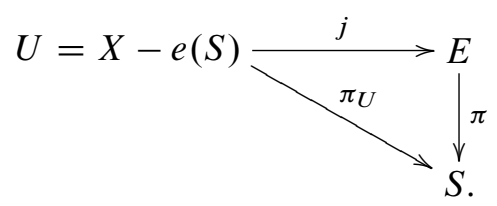

The restriction of $\mathscr{L}_{\log }$ to $U$ will be denoted by $\mathscr{L}^{\circ} g_{U}$.

Lemma 2.4 ([3], 1.2.8). The higher direct images of $\mathscr{L}^{\circ} g_{U}$ are

$$
R^{n} \pi_{U *} \mathscr{L}^{\circ} g_{U}= \begin{cases}0 & \text { if } n \neq 1, \\ \mathcal{I}(-1) & \text { if } n=1 .\end{cases}
$$


By this lemma, the edge morphism in the Leray spectral sequence for $R \pi_{U} *$ is an isomorphism $\operatorname{Ext}_{U}^{1}\left(\pi_{U}^{*} \mathcal{I}, \mathscr{L}_{\operatorname{og}}(1)\right) \simeq \operatorname{Hom}_{S}(\mathcal{I}, \mathcal{I})$.

Definition 2.5 ([3], 1.3.5.). The large elliptic polylogarithm

$$
\operatorname{Pol}_{E} \in \operatorname{Ext}_{U}^{1}\left(\pi_{U}^{*} \mathcal{I}, \mathscr{L} o g_{U}\right)
$$

is the extension class mapping to the identity map under the above isomorphism.

It is shown in Section 3.2.3 of [12] how to extend to $\mathcal{P o l}_{E}$ the action of $\pi^{*} \mathcal{R}$ on $\mathscr{L o g}_{U}$ and $\pi^{*} \mathcal{I}$ so that $\operatorname{Pol}_{E} \in \operatorname{Ext}_{U, \pi^{*} \mathcal{R}}^{1}\left(\pi_{U}^{*} \mathcal{I}, \mathscr{L} \operatorname{og}_{U}\right)$ is an extension class of $\pi^{*} \mathcal{R}$-modules.

2.1. Functoriality and invariance by torsion sections. Let $f: S^{\prime} \rightarrow S$ be a connected scheme over $S$ of characteristic zero. Form the cartesian square

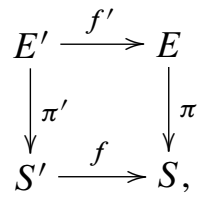

and let $e^{\prime}: S^{\prime} \rightarrow E^{\prime}$ be the unit section of $E^{\prime}$ and $U^{\prime}=E^{\prime}-e^{\prime}\left(S^{\prime}\right)$. We denote with superscripts ' the pro-sheaves $\mathcal{R}^{\prime}=e^{\prime *} \mathscr{L} g_{U^{\prime}}$ and $I^{\prime}=\operatorname{ker}\left(\mathcal{R}^{\prime} \rightarrow \mathbb{Q}_{p}\right)$ over $S^{\prime}$.

Lemma 2.6. We have a canonical isomorphism $\mathscr{L o g}_{E^{\prime}}=f^{\prime *} \mathscr{L}_{\text {og }}$ and a canonical isomorphism $\mathcal{R}^{\prime}=f^{*} \mathcal{R}$

Proof. For every integer $j \geq 0$, as $\left[p^{j}\right]$ is an étale cover, hence is proper, we have the proper base change canonical isomorphism $f^{\prime *}\left[p^{j}\right]_{*} \mathbb{Q}_{p}=\left[p^{j}\right]_{*}^{\prime} f^{\prime *} \mathbb{Q}_{p}=$ $\left[p^{j}\right]_{*}^{\prime} \mathbb{Q}_{p}$, where $\left[p^{j}\right]^{\prime}$ denotes the multiplication by $p^{j}$ on $E^{\prime}$. Using the above description of the logarithm pro-sheaf, we get the first announced canonical isomorphism. Now by functoriality

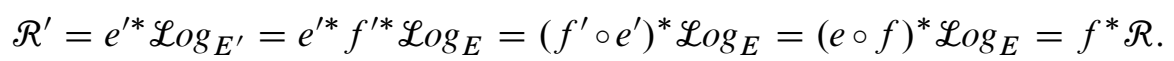

Note that the same argument shows that for any isogeny $f^{\prime}: E^{\prime} \rightarrow E$ over $S$ we also have the canonical isomorphism $\mathscr{L}^{\circ} g_{E^{\prime}}=f^{\prime *} \mathscr{L}_{\log }$. A consequence of this identity that will be useful in what follows is the invariance of the logarithm by translation by torsion sections.

Lemma 2.7. Let $t: S \rightarrow E$ be a torsion section. Then there is a canonical isomorphism

$$
t^{*} \mathscr{L}_{E}=e^{*} \mathscr{L} \operatorname{og}_{E}
$$


Proof. Let $n$ be the order of $t$ and denote by $[n]: E \rightarrow E$ the multiplication by $n$ over $S$. We have

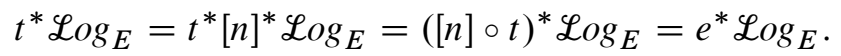

Lemma 2.8. There is a canonical isomorphism of extension of pro-sheaves $\mathcal{P o l}_{E^{\prime}}=$ $f^{\prime *} \mathcal{P o l}_{E}$

Proof. The functoriality of the logarithm (Lemma 2.6) gives rise to a commutative diagram

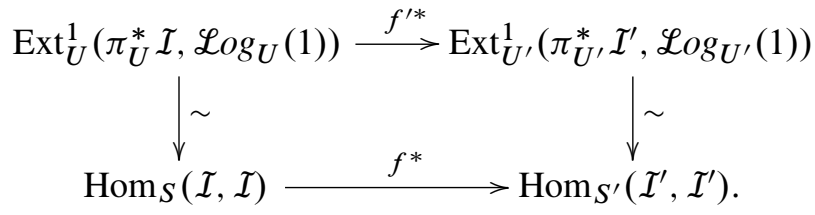

As the lower horizontal arrow maps the identity to the identity, we obtain the announced result.

2.2. Norm compatibility. This subsection is the most important for our following application: we recall the norm compatibility property of the large elliptic polylogarithm pro-sheaf.

Let us consider an arbitrary elliptic curve $\pi^{\prime}: E^{\prime} \rightarrow S$, with unit section $e^{\prime}: S^{\prime} \rightarrow$ $E^{\prime}$ and with complementary open $U^{\prime}=E^{\prime}-e^{\prime}\left(S^{\prime}\right)$. Let $f: E^{\prime} \rightarrow E$ be an isogeny, with kernel $Z$ and consider $\widetilde{U}=f^{-1}(U)$. We have a commutative diagram with cartesian squares:

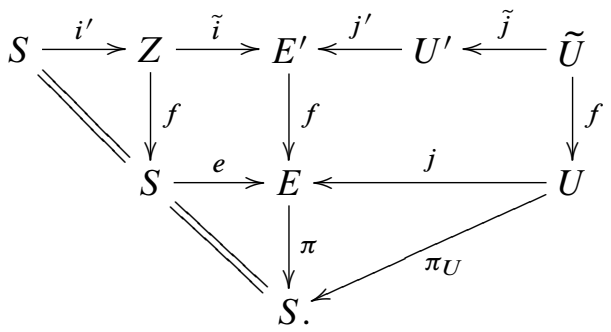

The adjunction map $\mathscr{L}_{\operatorname{og}_{U^{\prime}}}(1) \rightarrow \tilde{j}_{*} \tilde{j}^{*} \mathscr{L}_{o g_{U^{\prime}}}(1)$ of restriction to $\tilde{U}$ gives rise to a map

$$
\operatorname{Ext}_{U^{\prime}}^{1}\left(\pi_{U^{\prime}}^{*} \mathcal{I}, \mathscr{L}_{\log _{U^{\prime}}}(1)\right) \stackrel{\tilde{j}^{*}}{\longrightarrow} \operatorname{Ext}_{\widetilde{U}}^{1}\left(\pi_{U^{\prime}}^{*} \mathcal{I}, \tilde{j}_{*} \tilde{j}^{*} \mathscr{L}_{o g}(1)\right)
$$

Now by adjunction

$$
\begin{aligned}
& \operatorname{Ext}_{\widetilde{U}}^{1}\left(\pi_{U^{\prime}}^{*} \mathcal{I}, \tilde{j}_{*} \tilde{j}^{*} \mathscr{L}_{o g}(1)\right)=\operatorname{Ext}_{\widetilde{U}^{\prime}}^{1}\left(\tilde{j}^{*} \pi_{U^{\prime}}^{*} \mathcal{I}, \tilde{j}^{*} \mathscr{L o g}_{U^{\prime}}(1)\right) \\
& \quad=\operatorname{Ext}_{\widetilde{U}^{\prime}}^{1}\left(f^{*} \pi_{U}^{*} \mathcal{I}, \tilde{j}^{*} \mathscr{L}_{\log }(1)\right)=\operatorname{Ext}_{U}^{1}\left(\pi_{U}^{*} \mathcal{I}, f_{*} \tilde{j}^{*} \mathscr{L}_{\log }(1)\right) .
\end{aligned}
$$


By the functoriality of the logarithm $\mathscr{L}^{\circ} g_{U^{\prime}}=f^{*} \mathscr{L} g_{U}$, the right hand term of the last $\operatorname{Ext}^{1}$ can be written $f_{*} \tilde{j}^{*} \mathscr{L}_{o} g_{U^{\prime}}(1)=f_{*} f^{*} \mathscr{L} g_{U}(1)$. As $f$ is an étale cover we have the trace map tr: $f_{*} f^{*} \mathscr{L}_{\log }(1) \rightarrow \mathscr{L} g_{U}(1)$, so we finally obtain a norm morphism

$$
N_{f}=\operatorname{tr} \circ \tilde{j}^{*}: \operatorname{Ext}_{U^{\prime}}^{1}\left(\pi_{U^{\prime}}^{*} \mathcal{I}, \mathscr{L} o g_{U^{\prime}}(1)\right) \longrightarrow \operatorname{Ext}_{U}^{1}\left(\pi_{U}^{*} \mathcal{I}, \mathscr{L} o g_{U}(1)\right)
$$

Proposition 2.9 ([21], III, Chapter 5, Theorem 5.2 and [13], Proposition 2.2.1). For every isogeny $f$ we have

$$
N_{f}\left(\operatorname{Pol}_{U^{\prime}}\right)=\operatorname{Pol}_{U}
$$

2.3. Pull-backs along torsion sections. This subsection entirely relies on [12], Section 3.5.3. We wish to associate some absolute étale cohomology classes to pullbacks of the large elliptic polylogarithm along torsion sections.

This can be done in the following way. Let $\pi: E \rightarrow S$ be an elliptic curve and $t: S \rightarrow E$ be a non zero torsion section of $\pi$. Denote by $\mathscr{H}$ the relative Tate module $\underline{\operatorname{Hom}}\left(R^{1} \pi_{*} \mathbb{Q}_{p}, \mathbb{Q}_{p}\right)$, by Sym $\mathscr{H}=\bigoplus_{k} \operatorname{Sym}^{k} \mathscr{H}$ its symmetric algebra and by $\operatorname{Sym}^{\geq n} \mathscr{H} \subset \operatorname{Sym} \mathscr{H}$ the ideal $\bigoplus_{k \geq n} \operatorname{Sym}^{k} \mathscr{H}$. Identifying the symmetric algebra Sym $\mathscr{H}$ with the universal envelopping algebra $\mathcal{U}(\mathscr{H})$ of the abelian Lie algebra $\mathscr{H}$, we give to it the structure of a Hopf algebra. Denote by $\hat{U}(\mathscr{H})=\prod_{k \geq 0} \operatorname{Sym}^{k} \mathscr{H}$ the completion of $\mathcal{U}(\mathscr{H})$ along the augmentation ideal.

As the logarithm is translation invariant along $t$ (Lemma 2.7), we have $t^{*} \mathcal{P o l}_{E} \in$ $\operatorname{Ext}_{U}^{1}(\mathcal{I}, \mathcal{R}(1))$. Denote by $\mathcal{R}^{(n)}=e^{*} \mathscr{L} \operatorname{og}_{E}^{(n)}$. The pro-sheaf $\mathcal{R}=\left(\mathcal{R}^{(n)}\right)_{n}$ is a Hopf algebra ([3], 1.2.10 iv) and we have an isomorphism of Hopf algebras

$$
v^{n}: \operatorname{Sym} \mathscr{H} / \mathrm{Sym}^{\geq n+1} \mathscr{H} \stackrel{\sim}{\longrightarrow} \mathrm{Gr}^{\leq n} \mathcal{R}^{(n)}
$$

according to [loc. cit.], Proposition 1.2.6. By the structure theorem [4], chapitre II, paragraph 1 , no. 6 , the maps $v^{n}$ lift to an isomorphism of Hopf algebras

$$
v: \hat{U}(\mathscr{H}) \stackrel{\sim}{\longrightarrow} \mathcal{R} .
$$

Now consider the Koszul resolution

$$
0 \longrightarrow \bigwedge^{2} \mathscr{H} \otimes \mathcal{R}=\mathcal{R}(1) \mathscr{H} \otimes \mathcal{R} \stackrel{b}{\longrightarrow} \mathcal{I} \longrightarrow 0
$$

of the Lie algebra $\mathscr{H}$, where the first map is $(x \otimes y-y \otimes x) \otimes u \mapsto x \otimes y u-y \otimes x u$ and the second is $h \otimes u \mapsto h u$. By [12], Lemma 3.5.8, the map

$$
\begin{array}{r}
\operatorname{Ext}_{S}^{1}\left(\mathbb{Q}_{p}, \mathcal{R}(1)\right) \simeq \operatorname{Ext}_{S, \mathcal{R}}^{1}(\mathcal{R}, \mathcal{R}(1)) \stackrel{a}{\longrightarrow} \operatorname{Ext}_{S, \mathcal{R}}^{1}(\mathcal{I}, \mathcal{R}(1)) \\
\stackrel{b^{*}}{\longrightarrow} \operatorname{Ext}_{S, \mathcal{R}}^{1}(\mathcal{H} \otimes \mathcal{R}, \mathcal{R}(1))
\end{array}
$$


has a functorial splitting $\iota$. Composing with the projection induced by $\mathcal{R}(1) \rightarrow$ $\operatorname{Sym}^{k} \mathcal{H}(1)$, we get the absolute cohomology classes

$$
E_{t}^{k}=\iota\left(b^{*} t^{*} \mathcal{P o l}_{E}\right)^{k} \in \mathrm{H}^{1}\left(S, \operatorname{Sym}^{k} \mathscr{H}(1)\right) .
$$

The comparison of these classes with Beilinson's Eisenstein symbol ([2], Theorem 7.3) is given in [8], Theorem 2.2.4.

2.4. The compatibility between 2.2 and 2.3. Let $f: E^{\prime} \rightarrow E$ be an isogeny over $S$ and $t: S \rightarrow E$ be a torsion section. We assume that $f$ is trivial over $S$, or in other terms, that we have a cartesian square

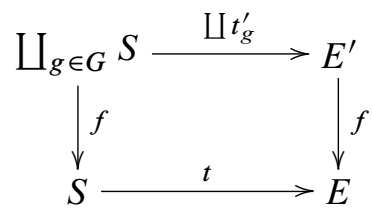

where $G$ is the Galois group of $f$. Therefore the $t_{g}^{\prime}$ are non zero torsion sections of $E^{\prime}$.

Lemma 2.10. In $\mathrm{H}^{1}\left(S, \operatorname{Sym}^{k} \mathscr{H}(1)\right)$ we have $E_{t}^{k}=\sum_{g \in G} E_{t_{g}^{\prime}}^{k}$.

Proof. By the norm compatibility of the polylogarithm (proposition 2.9) we have

$$
E_{t}^{k}=\iota\left(b^{*} t^{*} \mathcal{P o l}_{E}\right)^{k}=\iota\left(b^{*} t^{*} N_{f} \mathcal{P o l}_{E^{\prime}}\right)^{k} .
$$

Recall that the norm morphism $N_{f}$ (2.2) is defined by composing the trace map

$$
\operatorname{Ext}_{U}^{1}\left(\pi_{U}^{*} \mathcal{I}, f_{*} f^{*} \mathscr{L}_{o}(1)\right) \stackrel{\mathrm{tr}}{\longrightarrow} \operatorname{Ext}_{U}^{1}\left(\pi_{U}^{*} \mathcal{I}, \mathscr{L}_{0}(1)\right)
$$

with the restriction to the inverse image by $f$ of the complementary of the zero section of $E$

$$
\begin{aligned}
\operatorname{Ext}_{U^{\prime}}^{1}\left(\pi_{U^{\prime}}^{*} \mathcal{I}, \mathscr{L}_{\log }(1)\right) \stackrel{\tilde{j}_{U^{\prime}}^{*}}{\longrightarrow} \operatorname{Ext}_{\widetilde{U}^{1}}^{1}\left(\pi_{U^{\prime}}^{*} \mathcal{I}, \tilde{j}_{*} \tilde{j}^{*} \mathscr{L}_{\log _{U^{\prime}}}(1)\right) \\
=\operatorname{Ext}_{U}^{1}\left(\pi_{U}^{*} \mathcal{I}, f_{*} f^{*} \mathscr{L}_{\log }(1)\right)
\end{aligned}
$$

By the base change $t^{*} f_{*} f^{*}=\left(f_{*} t_{g}^{\prime *} f^{*}\right)_{g \in G}=\sum_{g \in G} t_{g}^{\prime *}$ we have $t^{*} f_{*} f^{*} \mathscr{L}_{o} g_{E}=$ $\sum_{g \in G} t_{g}^{\prime *} \mathscr{L}_{\log _{E^{\prime}}}$. As a consequence

$$
E_{t}^{k}=\iota\left(b^{*} t^{*} N_{f} \mathcal{P o l}_{E^{\prime}}\right)^{k}=\sum_{g \in G} \iota\left(b^{*} t_{g}^{\prime *} \mathcal{P o l}_{E}\right)^{k}=\sum_{g \in G} E_{t_{g}^{\prime}}^{k} .
$$




\section{The norm relations}

Let $N \geq 3$ be an integer and let $\mathrm{Y}(N)$ be the modular curve of level $N$ : it is a smooth affine connected curve over $\mathbb{Q}\left(\zeta_{N}\right)$ representing the functor on $\mathbb{Q}$-schemes associating to a $\mathbb{Q}$-scheme $S$ the set of isomorphism classes of triples $\left(E, e_{1}, e_{2}\right)$ where $\pi: E \rightarrow S$ is an elliptic curve over $S$ and $\left(e_{1}, e_{2}\right)$ is a basis of the $N$-torsion of $E$ (see [6], IV. 2, for details). The group $\mathrm{GL}_{2}(\mathbb{Z} / N)$ acts on $\mathrm{Y}(N)$ on the left: for $\sigma=\left(\begin{array}{ll}a & b \\ c & d\end{array}\right) \in \mathrm{GL}_{2}(\mathbb{Z} / N)$, define $\sigma \cdot\left(E, e_{1}, e_{2}\right)=\left(E, e_{1}^{\prime}, e_{2}^{\prime}\right)$ where

$$
\left(\begin{array}{l}
e_{1}^{\prime} \\
e_{2}^{\prime}
\end{array}\right)=\left(\begin{array}{ll}
a & b \\
c & d
\end{array}\right)\left(\begin{array}{l}
e_{1} \\
e_{2}
\end{array}\right) .
$$

For $M, N \geq 3$, the modular curves $\mathrm{Y}(M, N)$ are defined as follows: chose a common multiple $L$ of $M$ and $N$, define the group

$$
G=\left\{\left(\begin{array}{ll}
a & b \\
c & d
\end{array}\right) \in \mathrm{GL}_{2}(\mathbb{Z} / L) ; a \equiv 1(M), b \equiv 0(M), c \equiv 0(N), d \equiv 1(N)\right\}
$$

and

$$
\mathrm{Y}(M, N)=G \backslash \mathrm{Y}(L),
$$

which is independent of the choice of $L$. The $\mathbb{Q}$-scheme $\mathrm{Y}(M, N)$ represents the functor associating to a $\mathbb{Q}$-scheme $S$ the set of isomorphism classes of triples $\left(E, e_{1}, e_{2}\right)$ where $\pi: E \rightarrow S$ is an elliptic curve over $S$ and $e_{1}$ and $e_{2}$ are sections of $\pi$ of order $M$ and $N$ respectively and such that the map $\mathbb{Z} / M \times \mathbb{Z} / N \rightarrow E$ defined by $(a, b) \mapsto a e_{1}+b e_{2}$ is injective. The curves $\mathrm{Y}(N)$ and $\mathrm{Y}(M, N)$ carry a universal elliptic curve $E$ by their very definition.

For every two integers $N \mid N^{\prime}$ there is an étale cover $f_{N^{\prime} N}: \mathrm{Y}\left(N^{\prime}\right) \rightarrow \mathrm{Y}(N)$ sending the sections $\left(e_{1}, e_{2}\right)$ over $\mathrm{Y}\left(N^{\prime}\right)$ to $\left(\frac{N^{\prime}}{N} e_{1}, \frac{N^{\prime}}{N} e_{2}\right)$. As we are working with rational coefficients, the pull-back map

$$
\mathrm{H}^{1}\left(\mathrm{Y}(N), \mathrm{Sym}^{k} \mathscr{H}(1)\right) \stackrel{f_{N^{\prime} N}^{*}}{\longrightarrow} \mathrm{H}^{1}\left(\mathrm{Y}\left(N^{\prime}\right), \mathrm{Sym}^{k} \mathscr{H}(1)\right)
$$

is injective. We now define some cohomology classes in $\bigcup_{N} \mathrm{H}^{1}\left(\mathrm{Y}(N), \operatorname{Sym}^{k} \mathscr{H}(1)\right)$ as follows: let $(\alpha, \beta)$ be a non zero element of $(\mathbb{Q} / \mathbb{Z})^{2}=\bigcup_{N} \frac{1}{N} \mathbb{Z} / \mathbb{Z}$. Choose an integer $N$ such that $N \alpha=N \beta=0$, write $(\alpha, \beta)=\left(\frac{a}{N}, \frac{b}{N}\right) \in \frac{1}{N} \mathbb{Z} / \mathbb{Z}=\mathbb{Z} / N \mathbb{Z}$ and define the Eisenstein class

$$
E_{\alpha, \beta}^{k}=E_{\left(a e_{1}+b e_{2}\right)}^{k} \in \mathrm{H}^{1}\left(\mathrm{Y}(N), \operatorname{Sym}^{k} \mathscr{H}(1)\right),
$$

where $E_{\left(\alpha e_{1}+\beta e_{2}\right)}^{k}$ is the class (2.4). In the bigger space $\bigcup_{N} \mathrm{H}^{1}\left(\mathrm{Y}(N), \operatorname{Sym}^{k} \mathscr{H}(1)\right)$, the Eisenstein class does not depend on $N$. 
Lemma 3.1. Let $(\alpha, \beta) \in(\mathbb{Q} / \mathbb{Z})^{2}-\{0,0\}$.

(i) For any $\sigma \in \mathrm{GL}_{2}(\mathbb{Z} / N)$ we have

$$
\sigma^{*} E_{\alpha, \beta}^{k}=E_{\alpha^{\prime}, \beta^{\prime}}^{k}
$$

where $\left(\alpha^{\prime}, \beta^{\prime}\right)=(\alpha, \beta) \sigma$.

(2) (Distribution property.) For any non zero integer a we have

$$
E_{\alpha, \beta}^{k}=\sum_{\alpha^{\prime}, \beta^{\prime}} E_{\alpha^{\prime}, \beta^{\prime}}^{k}
$$

where $\left(\alpha^{\prime}, \beta^{\prime}\right)$ range over all elements of $(\mathbb{Q} / \mathbb{Z})^{2}$ such that a $\alpha^{\prime}=\alpha$ and a $\beta^{\prime}=\beta$.

Proof. (i) By functoriality of the splitting $\iota$ we have

$$
\begin{aligned}
\sigma^{*} E_{\alpha, \beta}^{k} & =\iota\left(\sigma^{*} b^{*}\left(\alpha e_{1}+\beta e_{2}\right)^{*} \mathcal{P} o l_{E}\right)^{k} \\
& =\iota\left(b^{*} \sigma^{*}\left(\alpha e_{1}+\beta e_{2}\right)^{*} \mathcal{P o l}_{E}\right)^{k} \\
& =\iota\left(b^{*}\left(\alpha^{\prime} e_{1}+\beta^{\prime} e_{2}\right)^{*} \mathcal{P o l}_{E}\right)^{k} .
\end{aligned}
$$

(ii) follows by taking $f=$ "multiplication by $a$ " in Lemma 2.10 .

In what follows, we have to consider $\mathbb{Q}_{p}$-adic étale sheaves on the Shimura varieties associated to finite dimensional algebraic representations of the group underlying the variety. With this end in view, let us recall the adelic description of the complex points of the modular curves $\mathrm{Y}(N)$. Let $Z(\mathbb{R})$ be the center of $\mathrm{GL}_{2}(\mathbb{R})$ and let $L_{\infty}^{\prime} \subset \mathrm{SL}_{2}(\mathbb{R})$ be a maximal compact subgroup. Write $L_{\infty}=Z(\mathbb{R}) L_{\infty}^{\prime} \subset \mathrm{GL}_{2}(\mathbb{R})$ for a maximal compact subgroup modulo the center. Consider the kernel $L(N)$ of the reduction modulo $N$ map $\mathrm{GL}_{2}(\widehat{\mathbb{Z}}) \rightarrow \mathrm{GL}_{2}(\mathbb{Z} / N \mathbb{Z})$. With our fixed complex embedding of $\mathbb{Q}\left(\zeta_{N}\right)$, we have the Shimura variety description of the modular curves

$$
\mathrm{Y}(N)(\mathbb{C})=\mathrm{GL}_{2}(\mathbb{Q}) \backslash\left(\mathrm{GL}_{2}(\mathbb{A}) / L(N) L_{\infty}\right) .
$$

Now according to (1.10) in [19], we can consider the $\mathbb{Q}_{p}$-adic étale sheaf on the $\mathrm{Y}(N)$ associated to the standard representation $\mathrm{V}$ of $\mathrm{GL}_{2}$ and we have a canonical isomorphism of $\mathbb{Q}_{p}$-adic sheaves $\mathrm{V}=\mathscr{H}$.

In order to give the definition of the cohomology classes we will consider, let us first remind about the weights of algebraic representations of $\mathrm{GL}_{2}$ and $\mathrm{GSp}_{4}$ : we choose a symplectic basis $\left(e_{1}, e_{2}, e_{3}, e_{4}\right)$ of $\left(V_{4}, \psi\right)$ such that

$$
\psi=\left(\begin{array}{cc}
0 & \mathrm{I}_{2} \\
-\mathrm{I}_{2} & 0
\end{array}\right)
$$


where $\mathrm{I}_{2}$ denotes the identity matrix of size 2 . Hence we have a symplectic isomorphism $V_{2} \oplus V_{2} \simeq V_{4}$ and the embedding $\iota: \mathrm{GL}_{2} \times_{\mathbb{G}_{m}} \mathrm{GL}_{2} \rightarrow \mathrm{GSp}_{4}$, the fibre product being over the determinant, given by

$$
\iota\left[\left(\begin{array}{ll}
a & b \\
c & d
\end{array}\right),\left(\begin{array}{ll}
a^{\prime} & b^{\prime} \\
c^{\prime} & d^{\prime}
\end{array}\right)\right]=\left(\begin{array}{cccc}
a & 0 & b & 0 \\
0 & a^{\prime} & 0 & b^{\prime} \\
c & 0 & d & 0 \\
0 & c^{\prime} & 0 & d^{\prime}
\end{array}\right)
$$

Let $\mathrm{T}_{2}$, resp. $\mathrm{T}_{4}$, be the diagonal maximal torus of $\mathrm{GL}_{2}$, resp. GSp $\mathrm{F}_{4}$. We have

$$
\mathrm{T}_{2}=\left\{\operatorname{diag}\left(\alpha, \alpha^{-1} v\right)=\left(\begin{array}{cc}
\alpha & 0 \\
0 & \alpha^{-1} v
\end{array}\right),(\alpha, v) \in \mathbb{G}_{m}^{2}\right\}
$$

and

$$
\mathrm{T}_{4}=\left\{\operatorname{diag}\left(\alpha_{1}, \alpha_{2}, \alpha_{1}^{-1} v, \alpha_{2}^{-1} v\right)=\left(\begin{array}{cccc}
\alpha_{1} & 0 & 0 & 0 \\
0 & \alpha_{2} & 0 & 0 \\
0 & 0 & \alpha_{1}^{-1} v & 0 \\
0 & 0 & 0 & \alpha_{2}^{-1} v
\end{array}\right),\left(\alpha_{1}, \alpha_{2}, v\right) \in \mathbb{G}_{m}^{3}\right\} .
$$

The group of characters $X^{*}\left(\mathrm{~T}_{n}\right)$ is identified to $\mathbb{Z}^{\frac{n}{2}+1}$ via $\lambda(k, t): \operatorname{diag}\left(\alpha, \alpha^{-1} v\right) \mapsto$ $\alpha_{1}^{k} v^{t}$ and $\lambda\left(k, k^{\prime}, t\right): \operatorname{diag}\left(\alpha_{1}, \alpha_{2}, \alpha_{1}^{-1} v, \alpha_{2}^{-1} v\right) \mapsto \alpha_{1}^{k} \alpha_{2}^{k^{\prime}} v^{t}$. Write $\rho_{1}=\lambda(1,-1,0)$ and $\rho_{2}=\lambda(0,2,0)$. Then the roots of $\mathrm{T}_{4}$ in $\mathrm{GSp}_{4}$ are $R=\left\{ \pm \rho_{1}, \pm \rho_{2}, \pm\left(\rho_{1}+\right.\right.$ $\left.\left.\rho_{2}\right), \pm\left(2 \rho_{1}+\rho_{2}\right)\right\}$ and the positive roots corresponding to the standard Borel are $R^{+}=\left\{\rho_{1}, \rho_{2}, \rho_{1}+\rho_{2}, 2 \rho_{1}+\rho_{2}\right\}$. For $\mathrm{T}_{4}$, dominants, resp. regular weights are the $\lambda\left(k, k^{\prime}, t\right)$ with $k \geq k^{\prime} \geq 0$, resp. $k>k^{\prime}>0$. Dominants, resp. regular, weights of $\mathrm{T}_{2}$ are the $\lambda(k, t)$ with $k \geq 0$, resp. $k>0$. The irreducible representation of $\mathrm{GL}_{2}$ of highest weight $\lambda(k, t)$ is the twisted symmetric product $\operatorname{Sym}^{k} \mathrm{~V} \otimes \operatorname{det}^{\otimes t}$.

Now let $k \geq k^{\prime} \geq 0$ be two integers and fix a finite dimensional algebraic representation $W^{k k^{\prime}}$ of $\mathrm{GSp}_{4}$ whose restriction $\iota^{*} W^{k} k^{\prime}$ contains the irreducible representation $\left(\operatorname{Sym}^{k} \mathrm{~V} \otimes \mathrm{Sym}^{k^{\prime}} \mathrm{V}\right) \otimes \operatorname{det}^{\otimes 3}$, that we will simply denote $\left(\operatorname{Sym}^{k} \mathrm{~V} \otimes \operatorname{Sym}^{k^{\prime}} \mathrm{V}\right)(3)$. Note that $W^{k k^{\prime}}$ has highest weight $\lambda\left(k, k^{\prime}, 3\right)$ hence is unique up to isomorphism. We will also denote by $W^{k} k^{\prime}$ the lisse étale sheaf over the Siegel threefolds corresponding to $W^{k k^{\prime}}$ according to [19], (1.10). To $\iota$ is associated a closed embedding, purely of codimension one

$$
\mathrm{Y}(N) \times_{\mathbb{Q}\left(\zeta_{N}\right)} \mathrm{Y}(N) \stackrel{\iota}{\rightarrow} \mathrm{S}(N) .
$$

in the Siegel modular threefold of level $N$. Then, the composition of the external cup-product

$$
\begin{aligned}
\mathrm{H}^{1}\left(\mathrm{Y}(N), \operatorname{Sym}^{k} \mathrm{~V}(1)\right) \otimes \mathrm{H}^{1}\left(\mathrm{Y}(N), \operatorname{Sym}^{k^{\prime}} \mathrm{V}(1)\right) \\
\stackrel{\sqcup}{\longrightarrow} \mathrm{H}^{2}\left(\mathrm{Y}(N) \times \times_{\mathbb{Q}\left(\zeta_{N}\right)} \mathrm{Y}(N),\left(\operatorname{Sym}^{k} \mathrm{~V} \otimes \operatorname{Sym}^{k^{\prime}} \mathrm{V}\right)(2)\right)
\end{aligned}
$$


of the morphism induced by the inclusion $\left(\operatorname{Sym}^{k} \mathrm{~V} \otimes \operatorname{Sym}^{k^{\prime}} \mathrm{V}\right)(2) \subset \iota^{*} W^{k k^{\prime}}(-1)$ and of the Gysin morphism

$$
\mathrm{H}^{2}\left(\mathrm{Y}(N) \times_{\mathbb{Q}\left(\zeta_{N}\right)} \mathrm{Y}(N), \iota^{*} W^{k k^{\prime}}(-1)\right) \stackrel{\iota^{*}}{\longrightarrow} \mathrm{H}^{4}\left(\mathrm{~S}(N), W^{k k^{\prime}}\right)
$$

is a morphism

$$
\mathrm{H}^{1}\left(\mathrm{Y}(N), \operatorname{Sym}^{k} \mathrm{~V}(1)\right) \otimes \mathrm{H}^{1}\left(\mathrm{Y}(N), \operatorname{Sym}^{k^{\prime}} \mathrm{V}(1)\right) \longrightarrow \mathrm{H}^{4}\left(\mathrm{~S}(N), W^{k k^{\prime}}\right) .
$$

For any $N \geq 3$, we denote by

$$
\left.E_{N}^{k k^{\prime}}=\iota_{*}\left(E_{1 / N, 0}^{k} \sqcup E_{0,1 / N}^{k^{\prime}}\right) \in \mathrm{H}^{4}(\mathrm{~S}(N)), W^{k k^{\prime}}\right)
$$

the image of $E_{1 / N, 0}^{k} \otimes E_{0,1 / N}^{k^{\prime}}$ under this morphism.

The proof of the following proposition is very similar to the one of [10], Proposition 2.3 in the case of $\mathrm{GL}_{2}$.

Proposition 3.2. For every two integers $N \mid N^{\prime}$ with the same prime factors the trace morphism $\mathrm{H}^{4}\left(\mathrm{~S}\left(N^{\prime}\right), W^{k k^{\prime}}\right) \rightarrow \mathrm{H}^{4}\left(\mathrm{~S}(N), W^{k k^{\prime}}\right)$ sends $E_{N^{\prime}}^{k k^{\prime}}$ to $d_{N, N^{\prime}}^{2} E_{N}^{k k^{\prime}}$ where $d_{N, N^{\prime}}=\left(N^{\prime} / N\right)^{2}$ is the degree of the étale cover $\mathrm{Y}\left(N, N^{\prime}\right) \rightarrow \mathrm{Y}(N)$.

Proof. The Gysin morphism and the trace are induced by the adjunction morphisms $\iota ! l^{!} \rightarrow 1$ and $f_{*} f^{*}=f ! f^{!} \rightarrow 1$ respectively, so by functoriality they commute. As a consequence it is enough to show that $E_{1 / N^{\prime}, 0}^{k} \sqcup E_{0,1 / N^{\prime}}^{k^{\prime}}$ is mapped to $d_{N, N^{\prime}}^{2} E_{1 / N, 0}^{k} \sqcup$ $E_{0,1 / N}^{k^{\prime}}$ under the trace

$$
\begin{aligned}
& \mathrm{H}^{2}\left(\mathrm{Y}\left(N^{\prime}\right) \times \mathrm{Y}\left(N^{\prime}\right),\left(\operatorname{Sym}^{k} \mathrm{~V} \otimes \operatorname{Sym}^{k^{\prime}} \mathrm{V}\right)(2)\right) \\
& \quad \longrightarrow \mathrm{H}^{2}\left(\mathrm{Y}(N) \times \mathrm{Y}(N),\left(\operatorname{Sym}^{k} \mathrm{~V} \otimes \operatorname{Sym}^{k^{\prime}} \mathrm{V}\right)(2)\right)
\end{aligned}
$$

Denote by $p_{i}: \mathrm{Y}\left(N^{\prime}\right) \times \mathrm{Y}\left(N^{\prime}\right) \rightarrow \mathrm{Y}\left(N^{\prime}\right)$ the $i$-th projection. In terms of the usual cup-product, the external cup product is given by

$$
E_{1 / N^{\prime}, 0}^{k} \sqcup E_{0,1 / N^{\prime}}^{k^{\prime}}=p_{1}^{*} E_{1 / N^{\prime}, 0}^{k} \cup p_{2}^{*} E_{0,1 / N^{\prime}}^{k^{\prime}} .
$$

Then, denoting by $U$ the Galois group of $f_{N^{\prime} N}: \mathrm{Y}\left(N^{\prime}\right) \rightarrow \mathrm{Y}(N)$, we have

$$
\begin{aligned}
\operatorname{tr}\left(E_{1 / N^{\prime}, 0}^{k} \sqcup E_{0,1 / N^{\prime}}^{k^{\prime}}\right) & =\operatorname{tr}\left(p_{1}^{*} E_{1 / N^{\prime}, 0}^{k} \cup p_{2}^{*} E_{0,1 / N^{\prime}}^{k^{\prime}}\right) \\
& =\sum_{\sigma \times \sigma^{\prime} \in U \times U}\left(\sigma \times \sigma^{\prime}\right)^{*}\left(p_{1}^{*} E_{1 / N^{\prime}, 0}^{k} \cup p_{2}^{*} E_{0,1 / N^{\prime}}^{k^{\prime}}\right) \\
& \left.\left.=\sum_{\sigma \times \sigma^{\prime} \in U \times U}\left[\left(\sigma \times \sigma^{\prime}\right)^{*} p_{1}^{*} E_{1 / N^{\prime}, 0}^{k}\right)\right] \cup\left[\left(\sigma \times \sigma^{\prime}\right)^{*} p_{2}^{*} E_{0,1 / N^{\prime}}^{k^{\prime}}\right)\right]
\end{aligned}
$$




$$
\begin{aligned}
& =\sum_{\sigma \times \sigma^{\prime} \in U \times U}\left(p_{1}^{*} \sigma^{*} E_{1 / N^{\prime}, 0}^{k}\right) \cup\left(p_{2}^{*} \sigma^{\prime *} E_{0,1 / N^{\prime}}^{k^{\prime}}\right) \\
& \left.=p_{1}^{*}\left(\sum_{\sigma \in U} \sigma^{*} E_{1 / N^{\prime}, 0}^{k}\right)\right) \cup p_{2}^{*}\left(\sum_{\sigma \in U^{\prime}} \sigma^{\prime *} E_{0,1 / N^{\prime}}^{k^{\prime}}\right) \\
& =\operatorname{tr}\left(E_{1 / N^{\prime}, 0}^{k}\right) \sqcup \operatorname{tr}\left(E_{0,1 / N^{\prime}}^{k^{\prime}}\right)
\end{aligned}
$$

and we are led to show that $\operatorname{tr}\left(E_{1 / N^{\prime}, 0}^{k}\right)=d_{N, N^{\prime}} E_{1 / N, 0}^{k}$.

The étale cover $f_{N^{\prime} N}: \mathrm{Y}\left(N^{\prime}\right) \rightarrow \mathrm{Y}(N)$ factors as $\mathrm{Y}\left(N^{\prime}\right) \rightarrow \mathrm{Y}\left(N, N^{\prime}\right) \rightarrow \mathrm{Y}(N)$. By (3.1) the Galois group of the first cover is

$$
H=\left\{\left(\begin{array}{ll}
a & b \\
0 & 1
\end{array}\right) \in \mathrm{GL}_{2}\left(\mathbb{Z} / N^{\prime}\right) ; a \equiv 1(N), b \equiv 0(N)\right\} .
$$

Write $\alpha=N^{\prime} / N$. As $N^{\prime}$ and $N$ have the same prime factors, for any $(x, y) \in$ $(\mathbb{Z} / \alpha)^{2}$ we can fix an element $s_{x, y} \in \mathrm{GL}_{2}\left(\mathbb{Z} / N^{\prime}\right)$ of the form $\left(\begin{array}{c}1+N u N^{N v} \\ 0\end{array}\right)$ with $u \equiv x(\alpha)$ and $v \equiv y(\alpha)$ and $H=\left\{s_{x, y} ;(x, y) \in(\mathbb{Z} / \alpha)^{2}\right\}$. Hence the trace map $\mathrm{H}^{1}\left(\mathrm{Y}\left(N^{\prime}\right), \operatorname{Sym}^{k} \mathrm{~V}(1)\right) \rightarrow \mathrm{H}^{1}\left(\mathrm{Y}\left(N, N^{\prime}\right), \operatorname{Sym}^{k} \mathrm{~V}(1)\right)$ sends $E_{1 / N^{\prime}, 0}^{k}$ to

$$
\sum_{(x, y) \in(\mathbb{Z} / \alpha)^{2}} s_{(x, y)}^{*} E_{1 / N^{\prime}, 0}^{k}=\sum_{(x, y) \in(\mathbb{Z} / \alpha)^{2}} E_{1 / N^{\prime}+x / \alpha, y / \alpha}^{k}=E_{1 / N, 0}^{k},
$$

the first and the second equality follow from Lemma 3.1 (i) and (ii) respectively. Now consider the second étale cover $g: \mathrm{Y}\left(N, N^{\prime}\right) \rightarrow \mathrm{Y}(N)$ factorizing $f_{N^{\prime}, N}$. In $\mathrm{H}^{1}\left(\mathrm{Y}\left(N, N^{\prime}\right), \operatorname{Sym}^{k} \mathrm{~V}(1)\right)$ we have $E_{1 / N, 0}^{k}=g^{*} E_{1 / N, 0}^{k}$ hence the trace map $\mathrm{H}^{1}\left(\mathrm{Y}\left(N, N^{\prime}\right), \operatorname{Sym}^{k} \mathrm{~V}(1)\right) \rightarrow \mathrm{H}^{1}\left(\mathrm{Y}(N), \operatorname{Sym}^{k} \mathrm{~V}(1)\right)$ sends $E_{1 / N, 0}^{k}$ to $d_{N, N^{\prime}} E_{1 / N, 0}^{k}$.

Now fix an integer $N$ and define

$$
\mathcal{E}_{N p^{t}}^{k k^{\prime}}=\frac{1}{\prod_{i=1}^{t} d_{N p^{i-1}, N p^{i}}^{2}} E_{N p^{t}}^{k k^{\prime}} \in \mathrm{H}^{4}\left(\mathrm{~S}\left(N p^{t}\right), W^{k k^{\prime}}\right)
$$

where $d_{N p^{i-1}, N p^{i}}=p^{2}$ is the degree of the cover $\mathrm{Y}\left(N p^{i-1}, N p^{i}\right) \rightarrow \mathrm{Y}\left(N p^{i-1}\right)$.

Corollary 3.3. The class $\mathcal{E}_{N p^{t}}^{k k^{\prime}}$ is mapped to $\mathcal{E}_{N p^{t-1}}^{k k^{\prime}}$ under the norm map

$$
\mathrm{H}^{4}\left(\mathrm{~S}\left(N p^{t}\right), W^{k k^{\prime}}\right) \longrightarrow \mathrm{H}^{4}\left(\mathrm{~S}\left(N p^{t-1}\right), W^{k k^{\prime}}\right)
$$

\section{The final result}

This section heavily relies on the computations in Section 2.2 of [15]. We wish to show that our classes define a norm compatible system in the projective limit of Galois 
cohomology groups

$$
\lim _{t \geq 1} \mathrm{H}^{1}\left(\mathbb{Q}\left(\zeta_{N p^{t}}\right), \mathrm{H}^{3}\left(\mathrm{~S}\left(N p^{t}\right) \otimes \overline{\mathbb{Q}}, W^{k k^{\prime}}\right)\right)
$$

as predicted by the conjecture of Perrin-Riou. But let us first recall how the conjecture of Perrin-Riou associates a $p$-adic $L$-function to such a norm compatible system of Galois cohomology classes: $p$-adic $L$-functions live in a certain ring $\mathbb{H}_{\infty}$ which we need to introduce first. Let $G_{n}=\operatorname{Gal}\left(\mathbb{Q}\left(\zeta_{p^{n}}\right) / \mathbb{Q}\right)$ and $G_{\infty}=\lim _{n} G_{n}$. The cyclotomic character gives an isomorphism $\kappa: G_{\infty} \simeq \mathbb{Z}_{p}^{\times}$. Let $\Delta$ be the maximal finite subgroup of $G_{\infty}$, then we have the decomposition $G_{\infty}=\Delta \times G_{\infty}^{1}$ where $G_{\infty}^{1}=1+p \mathbb{Z}_{p} \subset \mathbb{Z}_{p}^{\times}$. Let $u$ be a topological generator of $G_{\infty}^{1}$. The Iwasawa algebra $\mathbb{Z}_{p}\left[\left[G_{\infty} \rrbracket\right]\right.$ is identified with the ring $\mathbb{Z}_{p}[\Delta][[u-1]]$ of formal power series over the group algebra $\mathbb{Z}_{p}[\Delta]$ in one variable $u-1$. For $h \geq 1$, let

$$
\begin{array}{r}
\mathbb{H}_{h}=\left\{\sum_{n \geq 0, \sigma \in \Delta} c_{n, \sigma} \sigma(u-1)^{n} \in \mathbb{Q}_{p}[\Delta][u-1] ; \lim _{n}\left|c_{n, \sigma}\right|_{p} n^{-h}=0,\right. \\
\text { for all } \sigma \in \Delta\}
\end{array}
$$

where ||$_{p}$ denotes the multiplicative valuation of $\mathbb{Q}_{p}$ normalized by $|p|_{p}=\frac{1}{p}$. Then

$$
\mathbb{Z}_{p}\left[\left[G_{\infty}\right]\right] \mathbb{H}_{1} \subset \mathbb{H}_{2} \subset \cdots .
$$

Define $\mathbb{H}_{\infty}=\bigcup_{h} \mathbb{H}_{h}$. Then $\mathbb{H}_{\infty}$ is a ring since $\mathbb{H}_{i} \mathbb{H}_{j} \subset \mathbb{H}_{i+j}$ for any $i, j \geq 1$. For any continuous character $\chi$ of $G_{\infty}$ we have a ring homomorphism $\mathbb{H}_{\infty} \rightarrow \overline{\mathbb{Q}}_{p}$ defined by

$$
\sum_{n \geq 0, \sigma \in \Delta} c_{n, \sigma} \sigma(u-1)^{n} \longmapsto \sum_{n \geq 0, \sigma \in \Delta} c_{n, \sigma} \chi(\sigma)(\chi(u)-1)^{n} .
$$

In the following we consider the usual Fontaine rings $\mathrm{B}_{\text {crys }} \subset \mathrm{B}_{d R}$ : recall that they have a continuous action of $\operatorname{Gal}\left(\overline{\mathbb{Q}}_{p} / \mathbb{Q}_{p}\right)$ and that $\mathrm{B}_{\text {crys }}$ has a Frobenius $\phi$ commuting with the Galois action. Given a $p$-adic Galois representation $M$, let $D_{d R}(M)=$ $\left(M \otimes \mathrm{B}_{d R}\right)^{\mathrm{Gal}\left(\overline{\mathbb{Q}}_{p} / \mathbb{Q}_{p}\right)}$ and let $D_{\text {crys }}(M)=\left(M \otimes \mathrm{B}_{\text {crys }}\right)^{\mathrm{Gal}\left(\overline{\mathbb{Q}}_{p} / \mathbb{Q}_{p}\right)}$. We say that $M$ is a de Rham representation of $\operatorname{Gal}\left(\overline{\mathbb{Q}}_{p} / \mathbb{Q}_{p}\right)$ if $\operatorname{dim}_{\mathbb{Q}_{p}} D_{d R}(M)=\operatorname{dim}_{\mathbb{Q}_{p}} M$.

Theorem 4.1 (Perrin-Riou, see [10], Theorem 16.4). Let $M$ be a p-adic representation of $\operatorname{Gal}\left(\overline{\mathbb{Q}}_{p} / \mathbb{Q}_{p}\right)$, i.e., a finite dimensional $\mathbb{Q}_{p}$-vector space endowed with a continuous $\mathbb{Q}_{p}$-linear action of $\operatorname{Gal}\left(\overline{\mathbb{Q}}_{p} / \mathbb{Q}_{p}\right)$. Assume $M$ is de Rham. Let $\eta \in$ $D_{\text {crys }}\left(M^{*}(1)\right)$ where $M^{*}$ is the dual Galois representation. Then there exists a unique homomorphism

$$
\mathscr{L}_{\eta}: \lim _{n} \mathrm{H}^{1}\left(\mathbb{Q}_{p}\left(\zeta_{p^{n}}\right), M\right) \longrightarrow \mathbb{H}_{\infty}
$$

having the following properties for any integer $r \geq 1$. 
(i) Let $n \geq 1$ and let $\chi: G_{n} \rightarrow \overline{\mathbb{Q}}_{p}^{\times}$be a homomorphism which does not factor through $G_{n-1}$. Then for any $x \in \lim _{n} \mathrm{H}^{1}\left(\mathbb{Q}_{p}\left(\zeta_{p^{n}}\right), M\right)$, we have

$$
\mathscr{L}_{\eta}(x)\left(\kappa^{r} \chi^{-1}\right)=(r-1) ! G\left(\chi, \zeta_{p^{n}}\right)^{-1} \sum_{\sigma \in G_{n}} \chi(\sigma)\left[\sigma\left(\exp ^{*}\left(x_{-r, n}\right)\right),\left(p^{-r} \phi\right)^{-n}(\eta)\right] .
$$

Here $G\left(\chi, \zeta_{p^{n}}\right)$ denotes the usual Gauss sum, $\exp ^{*}$ denotes the dual exponential map ([10], 9.3), $x_{-r, n}$ denotes the image of $x$ under the composite

$$
\lim _{n} \mathrm{H}^{1}\left(\mathbb{Q}_{p}\left(\zeta_{p^{n}}\right), M\right) \rightarrow \lim _{n} \mathrm{H}^{1}\left(\mathbb{Q}_{p}\left(\zeta_{p^{n}}\right), M(-r)\right) \rightarrow \mathrm{H}^{1}\left(\mathbb{Q}_{p}\left(\zeta_{p^{n}}\right), M(-r)\right)
$$

where the first arrow is the product with $\left(\left(\zeta_{p^{j}}\right)^{\otimes(-r)}\right)_{j \geq 1}$ and the second arrow is the canonical projection (so $\exp ^{*}\left(x_{-r, n}\right)$ is an element of $\mathbb{Q}\left(\zeta_{p^{n}}\right) \otimes D_{d R}(M(-r))=$ $\left.\mathbb{Q}\left(\zeta_{p^{n}}\right) \otimes D_{d R}(M)\right),[$,$] is the canonical pairing$

$$
\left(\mathbb{Q}\left(\zeta_{p^{j}}\right) \otimes D_{d R}(M)\right) \times D_{\text {crys }}\left(M^{*}(1)\right) \rightarrow \overline{\mathbb{Q}}_{p}
$$

induced by $D_{d R}(M) \times D_{\text {crys }}\left(M^{*}(1)\right) \rightarrow \overline{\mathbb{Q}}_{p}$ and $\phi$ is the Frobenius.

(2) Assume $\eta=\left(1-p^{-r} \phi\right) \eta^{\prime}$ with $\eta^{\prime} \in D_{\text {crys }}\left(M^{*}(1)\right)$. Then for any $x \in$ $\lim _{n} \mathrm{H}^{1}\left(\mathbb{Q}_{p}\left(\zeta_{p^{n}}\right), M\right)$,

$$
\mathscr{L}_{\eta}(x)\left(\kappa^{r}\right)=(r-1) !\left[\exp ^{*}\left(x_{-r, 0}\right),\left(1-p^{r-1} \phi^{-1}\right) \eta^{\prime}\right] .
$$

According to this theorem, once the norm compatible system $x$ is constructed, one has the $p$-adic $L$-function $\mathscr{L}_{\eta}(x) \in \mathbb{H}_{\infty}$. Then the difficult part is to show that this $p$-adic $L$-function interpolates the special values of the usual $L$-function. This will be carried over in a forthcoming paper by the explicit computation of the image of our norm compatible system under the dual exponential, result which is also expected to yield the non-vanishing of our classes, which is not known for the moment.

In order to obtain first Galois cohomology classes from our elements, we would like to show that the rank four étale cohomology $\mathrm{H}^{4}\left(\mathrm{~S}(N) \otimes \overline{\mathbb{Q}}, W^{k} k^{\prime}\right)$ has no invariants under the absolute Galois group of $\mathbb{Q}\left(\zeta_{N}\right)$, and then invoke the HochschildSerre spectral sequence. This relies on the following vanishing theorem of Saper: let $G$ be a connected reductive group over $\mathbb{Q}$, let $K_{\infty}$ be a maximal compact subgroup of $G(\mathbb{R})$, let $A_{G}$ be the identity component of a maximal $\mathbb{Q}$-split torus in the center of $G$ and let $\Gamma \subset G(\mathbb{Q})$ be an arithmetic subgroup. Write $D=G(\mathbb{R}) / K_{\infty} A_{G}$ and $X=\Gamma \backslash D$.

Theorem 4.2 ([20], Theorem 5). Assume $X$ is an arithmetic quotient of a Hermitian symmetric space of dimension $d$. Let $E$ be an algebraic irreducible representation of $G$ with regular highest weight. Then the singular cohomology $\mathrm{H}^{i}(S, E)$ with coefficients in $E$ vanishes for $i<\frac{d}{2}$. 
As this theorem is stated for arithmetic quotients of Hermitian symmetric spaces, let us recall how the Siegel threefolds $\mathrm{S}(N)(\mathbb{C})$ are a disjoint union of such: let $\mathscr{H}_{+}$ be Siegel upper half-plane

$$
\mathscr{H}_{+}=\left\{\left.\Omega \in \mathrm{M}_{2}(\mathbb{C})\right|^{t} \Omega=\Omega, \operatorname{Im} \Omega \gg 0\right\}
$$

of complex symmetric matrices of size two whose imaginary part is positive definite. It is known that $\mathscr{H}_{+}$is a Hermitian symmetric space. The symplectic group $\operatorname{Sp}_{4}(\mathbb{R})$ acts transitively on the left on $\mathscr{H}_{+}$via

$$
\left(\begin{array}{ll}
A & B \\
C & D
\end{array}\right) \cdot \Omega=(A \Omega+B)(C \Omega+D)^{-1}
$$

Let $\Gamma(N)=\operatorname{ker}\left(\operatorname{Sp}_{4}(\mathbb{Z}) \rightarrow \mathrm{Sp}_{4}(\mathbb{Z} / N \mathbb{Z})\right) \subset \mathrm{Sp}_{4}(\mathbb{R})$. The stabilizer of the matrix $i \mathrm{I}_{2}$ is a maximal compact subgroup of $\mathrm{Sp}_{4}(\mathbb{R})$. As the center of $\mathrm{Sp}_{4}$ is $\left\{ \pm \mathrm{I}_{4}\right\}$, note that $\mathscr{H}_{+}$is of the shape $D$ as above. According to [14], Proposition 3.2, we have an isomorphism of complex analytic varieties

$$
\mathrm{S}(N)(\mathbb{C})=\coprod_{(\mathbb{Z} / N \mathbb{Z})^{\times}} \Gamma(N) \backslash \mathscr{H}_{+} .
$$

We also have a similar statement for the modular curves $\mathrm{Y}(N)$ when the Siegel upper half-space is replaced by the Poincaré upper half-space.

The proof of the following Proposition 4.4 also relies on the main theorem of [19] that we are going to explain now in the needed particular case of $\mathrm{GSp}_{4}$.

\subsection{Higher direct images of $\boldsymbol{p}$-adic sheaves in the Baily-Borel compactification.}

The boundary of the Baily-Borel compactification of a Shimura variety associated to a group $G$ is stratified by (finite quotients of) Shimura varieties associated to the Levi subgroups of $G$. The main result of [19], that is stated at the end of this section, describes the restriction to a stratum of the higher direct image in the Baily-Borel compactification of the $p$-adic étale sheaf associated to an algebraic representation of $G$.

Let us first describe the construction of the Baily-Borel compactification of the Siegel threefolds. We will need the notion of pure and mixed Shimura datum for which we refer the reader to [18], 2. Consider the pure Shimura datum $\left(\mathrm{GSp}_{4}, \mathrm{GSp}_{4}(\mathbb{R}) / K_{\infty}\right)$ associated to the symplectic group $\mathrm{GSp}_{4}$. For every maximal parabolic subgroup $Q \subset \mathrm{GSp}_{4}$ there exists a normal subgroup $P_{1} \subset Q$ underlying a mixed Shimura datum $\left(P_{1}, \mathcal{X}_{1}\right)$ ([loc. cit.], 4.11), called a rational boundary component of $\left(\mathrm{GSp}_{4}, \mathrm{GSp}_{4}(\mathbb{R}) / K_{\infty}\right)$. Let $W_{1}$ be the unipotent radical of $P_{1}$ and let $q: P_{1} \rightarrow G_{1}=P_{1} / W_{1}$ be the projection on the Levi. Denote by $\left(G_{1}, \mathscr{H}_{1}\right)$ the quotient pure Shimura datum $\left(P_{1}, X_{1}\right) / W_{1}$ ([loc. cit.], Proposition 2.9). There are 
two conjugacy classes of maximal parabolic subgroups of $\mathrm{GSp}_{4}$, namely the ones of

$$
\begin{aligned}
& Q^{0}=W^{0} \rtimes\left(\mathbb{G}_{m} \times \mathrm{GL}_{2}\right)=\left\{\left(\begin{array}{cc}
\alpha A & A . M \\
0 & { }^{t} A^{-1}
\end{array}\right), \alpha \in \mathbb{G}_{m}, A \in \mathrm{GL}_{2},{ }^{t} M=M\right\}, \\
& Q^{1}=W^{1} \rtimes\left(\mathrm{GL}_{2} \times \mathbb{G}_{m}\right)=\left\{\left(\begin{array}{cccc}
\alpha & * & * & * \\
0 & a & * & b \\
0 & 0 & \beta & 0 \\
0 & c & * & d
\end{array}\right) \in \mathrm{GSp}_{4}, \alpha \beta=a d-b c\right\} .
\end{aligned}
$$

We also have, with obvious notations $P_{1}^{0}=W^{0} \rtimes \mathbb{G}_{m}, G_{1}^{0}=\mathbb{G}_{m}$ and $P_{1}^{1}=$ $W^{1} \rtimes \mathrm{GL}_{2}, G_{1}^{1}=\mathrm{GL}_{2}$ according to [loc. cit.], 4.25.

Let $\mathscr{H}^{*}=\bigsqcup_{\left(P_{1}, x_{1}\right)} \mathscr{H}_{1}$, the sum running over all rational boundary components of $\left(\mathrm{GSp}_{4}, \mathrm{GSp}_{4}(\mathbb{R}) / K_{\infty}\right)$. We endow $\mathscr{H}^{*}$ with the Satake topology ([loc. cit.], 6.2). Let

$$
\mathrm{S}(N)^{*}(\mathbb{C})=\operatorname{GSp}_{4}(\mathbb{Q}) \backslash\left(\mathscr{H}^{*} \times \mathrm{GSp}_{4}\left(\mathbb{A}_{f}\right) / K(N)\right) .
$$

Then $\mathrm{S}(N)^{*}(\mathbb{C})$ is the analytification of a normal projective $\mathbb{Q}\left(\zeta_{N}\right)$-scheme $\mathrm{S}(N)^{*}$ ([loc. cit.], 8.2). There is an open embedding $\mathrm{S}(N)(\mathbb{C}) \rightarrow \mathrm{S}(N)^{*}(\mathbb{C})$ which descends to an open embedding

$$
j: \mathrm{S}(N) \rightarrow \mathrm{S}(N)^{*} .
$$

For $n=0,1$ and every $g \in \operatorname{GSp}_{4}\left(\mathbb{A}_{f}\right)$ let $K_{1}^{n}=g K g^{-1} \cap P_{1}^{n}\left(\mathbb{A}_{f}\right)$ and

$$
\partial \mathrm{S}(N)_{n}(\mathbb{C})=G_{1}^{n}(\mathbb{Q}) \backslash\left(\mathscr{H}_{1}^{n} \times G_{1}^{n}\left(\mathbb{A}_{f}\right) / q\left(K_{1}^{n}\right)\right) .
$$

By [loc. cit.], Section 12.3 (b), the map $\partial \mathrm{S}(N)_{n}(\mathbb{C}) \rightarrow \mathrm{S}(N)^{*}(\mathbb{C})$ descends to

$$
i_{n}: \partial \mathrm{S}(N)_{n} \rightarrow \mathrm{S}(N)^{*} .
$$

Varying $n$ and $g$ we obtain a stratification of the boundary $\partial \mathrm{S}(N)=\mathrm{S}(N)^{*}-\mathrm{S}(N)$ by locally closed subschemes. For what follows, note that $\partial \mathrm{S}(N)_{n}$ is of dimension $n$.

Theorem 4.3 ([19], Theorem 4.2.1). Denote by $\mu$ the canonical construction of étale sheaves associated to representations of the group underlying a given Shimura variety ([loc. cit. $], 1.10)$. For $n=0,1$ there exists an arithmetic subgroup $H_{C} \subset Q^{n} / P_{1}^{n}(\mathbb{Q})$ such that

$$
i_{n}^{*} R^{m} j_{*} \mu\left(W^{k k^{\prime}}\right)=\bigoplus_{p+q=m} \mu\left(\mathrm{H}^{p}\left(H_{C}, \mathrm{H}^{q}\left(W^{n}, W^{k k^{\prime}}\right)\right)\right)
$$

Remark. In the proof of the following proposition we will quote the results of [15] where the computations are realized in the framework of mixed Hodge modules, rather than in the one of étale sheaves. There, Theorem 4.3 is replaced by the analogous theorem of Burgos-Wildeshaus (see [loc. cit.], Theorem 2.1). The result is exactly the same but on one shift that occurs in the graduation of the higher direct images due to the perverse t-structure: for example, as $\partial \mathrm{S}(N)_{0}$, resp. $\partial \mathrm{S}(N)_{1}$, is of 
codimension three, resp. two, in $\mathrm{S}(N)^{*}$, the higher direct image $i_{0}^{*} R^{m} j_{*} \mu\left(W^{k k^{\prime}}\right)$, resp. $i_{1}^{*} R^{m} j_{*} \mu\left(W^{k k^{\prime}}\right)$, in the framework of mixed Hodge modules corresponds to the higher direct image $i_{0}^{*} R^{m+3} j_{*} \mu\left(W^{k k^{\prime}}\right)$, resp. $i_{1}^{*} R^{m+2} j_{*} \mu\left(W^{k k^{\prime}}\right)$, in our étale setting.

\subsection{The weight computation}

Proposition 4.4. Assume $k>k^{\prime}>0$. Then the étale cohomology space $\mathrm{H}^{4}(\mathrm{~S}(N) \otimes$ $\left.\overline{\mathbb{Q}}, W^{k} k^{\prime}\right)$ has no weight zero. As a consequence, it has no invariants under the action of the absolute Galois group of $\mathbb{Q}\left(\zeta_{N}\right)$.

Proof. Let

$$
\mathrm{S}(N) \stackrel{j}{\longrightarrow} \mathrm{S}(N)^{*} \stackrel{i}{\longleftarrow} \mathrm{\partial S}(N)
$$

be the open embedding of $\mathrm{S}(N)$ into its Baily-Borel compactification and the reduced closed imbedding of the boundary. We have an exact sequence of Galois modules

$$
\begin{aligned}
\mathrm{H}_{c}^{4}\left(\mathrm{~S}(N) \otimes \overline{\mathbb{Q}}, W^{k k^{\prime}}\right) \longrightarrow & \mathrm{H}^{4}\left(\mathrm{~S}(N) \otimes \overline{\mathbb{Q}}, W^{k k^{\prime}}\right) \\
& \longrightarrow \mathrm{H}^{4}\left(\partial \mathrm{S}(N) \otimes \overline{\mathbb{Q}}, i^{*} R j_{*} W^{k k^{\prime}}\right) .
\end{aligned}
$$

With our assumption on the weight $k>k^{\prime}>0$, we can apply Theorem 4.2, which, together with Poincaré duality and the comparison theorem between étale and singular cohomology, shows the vanishing of the space $\mathrm{H}_{c}^{4}\left(\mathrm{~S}(N) \otimes \overline{\mathbb{Q}}, W^{k k^{\prime}}\right)$. So it is enough to show that $\mathrm{H}^{4}\left(\partial \mathrm{S}(N) \otimes \overline{\mathbb{Q}}, i^{*} R j_{*} W^{k k^{\prime}}\right)$ has no weight zero. Now let

$$
\partial \mathrm{S}(N)_{1} \stackrel{i_{1}}{\longrightarrow} \partial \mathrm{S}(N) \stackrel{i_{0}}{\longleftarrow} \partial \mathrm{S}(N)_{0}
$$

be the open embedding of the strata of dimension one and the reduced closed embedding of the strata of dimension zero respectively in the boundary. We have an exact sequence of Galois modules

$$
\begin{aligned}
\mathrm{H}_{c}^{4}\left(\partial \mathrm{S}(N)_{1} \otimes \overline{\mathbb{Q}}, i_{1}^{*} i^{*} R j_{*} W^{k k^{\prime}}\right) & \longrightarrow \mathrm{H}^{4}\left(\partial \mathrm{S}(N) \otimes \overline{\mathbb{Q}}, i^{*} R j_{*} W^{k k^{\prime}}\right) \\
& \longrightarrow \mathrm{H}^{4}\left(\partial \mathrm{S}(N)_{0} \otimes \overline{\mathbb{Q}}, i_{0}^{*} i^{*} R j_{*} W^{k k^{\prime}}\right)
\end{aligned}
$$

On the one hand, because $\partial \mathrm{S}(N)_{0}$ is of dimension zero we have

$$
\mathrm{H}^{4}\left(\partial \mathrm{S}(N)_{0} \otimes \overline{\mathbb{Q}}, i_{0}^{*} i^{*} R j_{*} W^{k k^{\prime}}\right)=\mathrm{H}^{0}\left(\partial \mathrm{S}(N)_{0} \otimes \overline{\mathbb{Q}}, i_{0}^{*} i^{*} R^{4} j_{*} W^{k k^{\prime}}\right)
$$

which has weight $>0$ according to [15], Lemma 2.5. On the other hand, we have the spectral sequence

$$
E_{2}^{p, q}=\mathrm{H}_{c}^{p}\left(\partial \mathrm{S}(N)_{1} \otimes \overline{\mathbb{Q}}, i_{1}^{*} i^{*} R^{q} j_{*} W^{k k^{\prime}}\right) \Longrightarrow \mathrm{H}_{c}^{p+q}\left(\partial \mathrm{S}(N)_{1} \otimes \overline{\mathbb{Q}}, i_{1}^{*} i^{*} R j_{*} W^{k} k^{\prime}\right) .
$$


Now Theorem 4.3 and Lemma 2.6 in [15] show that

$$
\begin{aligned}
i_{1}^{*} i^{*} R^{0} j_{*} W^{k k^{\prime}} & =i_{1}^{*} i^{*} R^{3} j_{*} W^{k k^{\prime}}=\operatorname{Sym}^{k^{\prime}} \mathrm{V}(3), \\
i_{1}^{*} i^{*} R^{1} j_{*} W^{k k^{\prime}} & =i_{1}^{*} i^{*} R^{2} j_{*} W^{k k^{\prime}}=\operatorname{Sym}^{k+1} \mathrm{~V}(3) .
\end{aligned}
$$

Recall that $\partial \mathrm{S}(N)_{1}$ is a disjoint union of modular curves. The fact that $\partial \mathrm{S}(N)_{1}$ is a non proper curve together with theorem 4.2 imply that the lisse étale sheaves above have cohomology concentrated in degree one. As a consequence our spectral sequence gives that

$$
\begin{aligned}
\mathrm{H}_{c}^{4}\left(\partial \mathrm{S}(N)_{1} \otimes \overline{\mathbb{Q}}, i_{1}^{*} i^{*} R j_{*} W^{k k^{\prime}}\right) & =\mathrm{H}_{c}^{1}\left(\partial \mathrm{S}(N)_{1} \otimes \overline{\mathbb{Q}}, i_{1}^{*} i^{*} R^{3} j_{*} W^{k} k^{\prime}\right) \\
& =\mathrm{H}_{c}^{1}\left(\partial \mathrm{S}(N)_{1} \otimes \overline{\mathbb{Q}}, \mathrm{Sym}^{k^{\prime}} \mathrm{V}(3)\right) .
\end{aligned}
$$

The lisse sheaf $\mathrm{V}$ has weight -1 , so the sheaf $\operatorname{Sym}^{k^{\prime}} \mathrm{V}(3)$ has weight $-k^{\prime}-6$ and the étale cohomology space $\mathrm{H}_{c}^{1}\left(\partial \mathrm{S}(N)_{1} \otimes \overline{\mathbb{Q}}, \mathrm{Sym}^{k^{\prime}} \mathrm{V}(3)\right)$ has weight smaller than $-k^{\prime}-6+1$ hence has no weight zero. As a consequence $\mathrm{H}^{4}\left(\partial \mathrm{S}(N) \otimes \overline{\mathbb{Q}}, i^{*} R j_{*} W^{k k^{\prime}}\right)$ has no weight zero and the proof is complete.

Corollary 4.5. Assume $k>k^{\prime}>0$. Then we have a canonical isomorphism

$$
\mathrm{H}^{1}\left(\mathbb{Q}\left(\zeta_{N}\right), \mathrm{H}^{3}\left(\mathrm{~S}(N) \otimes \overline{\mathbb{Q}}, W^{k k^{\prime}}\right)\right) \stackrel{\sim}{\longrightarrow} \mathrm{H}^{4}\left(\mathrm{~S}(N), W^{k k^{\prime}}\right) .
$$

Proof. Considering continuous Galois cohomology, we have the Hochschild-Serre spectral sequence

$$
E_{2}^{p, q}=\mathrm{H}^{p}\left(\mathbb{Q}\left(\zeta_{N}\right), \mathrm{H}^{q}\left(\mathrm{~S}(N) \otimes \overline{\mathbb{Q}}, W^{k k^{\prime}}\right)\right) \Longrightarrow \mathrm{H}^{p+q}\left(\mathrm{~S}(N), W^{k k^{\prime}}\right)
$$

([9], Corollary 3.4). By Theorem 4.2 and the comparison isomorphism between étale and singular cohomology, we have $\mathrm{H}^{q}\left(\mathrm{~S}(N) \otimes \overline{\mathbb{Q}}, W^{k k^{\prime}}\right)=0$ for $q<3$. Hence $E_{2}^{p, q}=0$ for $q<3$. As a consequence

$$
\begin{aligned}
E_{\infty}^{0,4}=\operatorname{ker} & \left(d: \mathrm{H}^{0}\left(\mathbb{Q}\left(\zeta_{N}\right), \mathrm{H}^{4}\left(\mathrm{~S}(N) \otimes \overline{\mathbb{Q}}, W^{k k^{\prime}}\right)\right)\right. \\
\longrightarrow & \left.\mathrm{H}^{2}\left(\mathbb{Q}\left(\zeta_{N}\right), \mathrm{H}^{3}\left(\mathrm{~S}(N) \otimes \overline{\mathbb{Q}}, W^{k k^{\prime}}\right)\right)\right)
\end{aligned}
$$

and $E_{\infty}^{1,3}=E_{2}^{1,3}$. But Proposition 4.4 asserts that $\mathrm{H}^{4}\left(\mathrm{~S}(N) \otimes \overline{\mathbb{Q}}, W^{k k^{\prime}}\right)$ has no weight zero. As a consequence $E_{\infty}^{0,4}=0$ and we have a canonical isomorphism

$$
\mathrm{H}^{1}\left(\mathbb{Q}\left(\zeta_{N}\right), \mathrm{H}^{3}\left(\mathrm{~S}(N) \otimes \overline{\mathbb{Q}}, W^{k k^{\prime}}\right) \stackrel{\sim}{\longrightarrow} \mathrm{H}^{4}\left(\mathrm{~S}(N), W^{k k^{\prime}}\right) .\right.
$$

From Corollary 3.3 and Corollary 4.5 we can now deduce the main result of this paper.

Proposition 4.6. Let $N \geq 1$ be an integer. Assume $k>k^{\prime}>0$. Then we have a norm compatible system

$$
\left(\varepsilon_{N p^{t}}^{k k^{\prime}}\right) \in \lim _{t \geq 1} \mathrm{H}^{1}\left(\mathbb{Q}\left(\zeta_{N p^{t}}\right), \mathrm{H}^{3}\left(\mathrm{~S}\left(N p^{t}\right) \otimes \overline{\mathbb{Q}}, W^{k k^{\prime}}\right)\right) .
$$




\section{References}

[1] M. Artin and B. Mazur, Etale homotopy. Reprint of the 1969 original, Lecture Notes in Math. 100, Springer-Verlag, Berlin 1986. Zbl 0182.26001 MR 0883959

[2] A. Beilinson, Higher regulators of modular curves. Contemp. Math. 55, Amer. Math. Soc., Providence, R.I., 1988. Zbl 0609.14006 MR 0862627

[3] A. Beilinson and A. Levin, The elliptic polylogarithm. In Motives (ed. by U. Jannsen, S. Kleiman and J.-P. Serre), Proc. Sympos. Pure Math. 55, Amer. Math. Soc., Providence, R.I., 1994, 123-190. Zbl 0817.14014 MR 1265553

[4] N. Bourbaki, Éléments de mathématique XXXVII, Groupes et algèbres de Lie. Chapitre II: Algèbres de Lie libres, Chapitre III: Groupes de Lie, Actualités Scientifiques et Industrielles 1349, Hermann, Paris 1972. Zbl 0244.22007 MR 0573068

[5] J. Coates and R. Sujatha, Cyclotomic fields and zeta values. Springer Monogr. Math., Springer-Verlag, Berlin 2006. Zbl 1100.11002 MR 2256969

[6] P. Deligne and M. Rapoport, Les schémas de modules des courbes elliptiques, Lecture Notes in Math. 349, Springer-Verlag, Berlin 1973. Zbl 0281.14010 MR 0337993

[7] T. Ekedahl, On the adic formalism. In The Grothendieck Festschrift, Vol. II, Progr. Math. 87, Birkhäuser, Boston, Mass., 1990, 197-218. Zbl 0821.14010 MR 1106899

[8] A. Huber and G. Kings, Degeneration of the $l$-adic Eisenstein classes and the elliptic polylog. Invent. Math. 135 (1999), 545-594. Zbl 0955.11027 MR 1669288

[9] U. Jannsen, Continuous étale cohomology. Math. Ann. 280 (1988), 207-245. Zbl 0649.14011 MR 0929536

[10] K. Kato, $p$-adic Hodge theory and values of zeta functions of modular forms. Astérisque 295 (2004), 117-290. Zbl 1142.11336 MR 2104361

[11] G. Kings, K-theory elements for the polylogarithm of abelian schemes. J. Reine Angew. Math. 517 (1999), 103-116. Zbl 1007.11043 MR 1728545

[12] G. Kings, The Tamagawa number conjecture for CM elliptic curves. Invent. Math. 143 (2001), 571-627. Zbl 1159.11311 MR 1817645

[13] G. Kings, A note on polylogarithms on curves and abelian schemes. Math. Z. 262 (2009), 527-537. Zbl 1191.11021 MR 2506305

[14] G. Laumon, Fonctions zêtas des variétés de Siegel. Astérisque 302 (2005), 1-66. Zbl 1097.11021 MR 2234859

[15] F. Lemma, Higher regulators, periods and special values of the degree four $L$-function of GSp(4). Preprint 2009; arXiv:0904.0757v2 [math.NT].

[16] D. Mumford and J. Fogarty, Geometric invariant theory. Ergeb. Math. Grenzgeb. 34, Springer-Verlag, Berlin 1982. Zbl 0504.14008 MR 0719371

[17] B. Perrin-Riou, Fonctions $L p$-adiques des représentations $p$-adiques. Astérisque 229 (1995). Zbl 0845.11040 MR 1327803

[18] R. Pink, Arithmetical compactifications of mixed Shimura varieties. Bonner Math. Schriften 209, Mathematisches Institut, Bonn 1990. Zbl 0748.14007 MR 1128753

[19] R. Pink, On $l$-adic sheaves on Shimura varieties and their higher direct images in the BailyBorel compactification. Math. Ann. 292 (1992), 197-240. Zbl 0748.14008 MR 1149032 
Vol. 85 (2010) A norm compatible system of Galois cohomology classes for GSp(4) 905

[20] L. Saper, $\mathscr{L}$-modules and a conjecture of Rapoport, Goresky, Mc Pherson. Astérisque 298 (2005), 319-333. Zbl 1083.11033 MR 2141706

[21] J. Wildeshaus, Realizations of polylogarithms. Lecture Notes in Math. 1650, Springer-Verlag, Berlin 1997. Zbl 0877.11001 MR 1482233

Received August 5, 2008

Francesco Lemma, 133, allée du Pré Corlet, 74370 Villaz, France

E-mail: francesco.lemma@polytechnique.org 